University of South Florida

DIGITAL COMMONS

Digital Commons @ University of

@ UNIVERSITY OF SOUTH FLORIDA

South Florida

October 2019

\title{
Experimental Results of a Model Reference Adaptive Control Approach on an Interconnected Uncertain Dynamical System
}

Kemberly Cespedes

University of South Florida

Follow this and additional works at: https://digitalcommons.usf.edu/etd

Part of the Mechanical Engineering Commons

\section{Scholar Commons Citation}

Cespedes, Kemberly, "Experimental Results of a Model Reference Adaptive Control Approach on an Interconnected Uncertain Dynamical System" (2019). USF Tampa Graduate Theses and Dissertations. https://digitalcommons.usf.edu/etd/8014

This Thesis is brought to you for free and open access by the USF Graduate Theses and Dissertations at Digital Commons @ University of South Florida. It has been accepted for inclusion in USF Tampa Graduate Theses and Dissertations by an authorized administrator of Digital Commons @ University of South Florida. For more information, please contact digitalcommons@usf.edu. 
Experimental Results of a Model Reference Adaptive Control Approach on an Interconnected Uncertain

Dynamical System

by

Kemberly Cespedes

A thesis submitted in partial fulfillment

of the requirements for the degree of

Master of Science in Mechanical Engineering

Department of Mechanical Engineering

College of Engineering

University of South Florida

Major Professor: Tansel Yucelen, Ph.D.

Rajiv Dubey, Ph.D.

Daniel Hess, Ph.D.

Date of Approval:

October 3, 2019

Keywords: Stability and performance guarantees, Interconnected dynamical systems, Benchmark mechanical system, Linear matrix inequalities, Physical interconnections

Copyright (C) 2019, Kemberly Cespedes 


\section{Dedication}

I am dedicating this thesis to my parents, Edward and Ana, who have always provided me with vast amounts of love, support and encouragement. This thesis is also dedicated to my best friends, Courtney and Hailey, for their emotional support, generosity and their sincerity. 


\section{Acknowledgments}

I would like to take this opportunity to express my gratitude to the people who helped me through the successful completion of this work. I would first like to express my sincere gratitude to my advisor, Dr. Tansel Yucelen, for his continued support, guidance, and immense knowledge. His enthusiasm and perseverance in doing research was both inspiring and motivational for me. I am particularly grateful to the other members of my thesis committee, Dr. Rajiv Dubey and Dr. Daniel Hess, for their time and helpful

comments. I would also wish to thank Dr. Rasim Guldiken for his immeasurable guidance through the graduate school process, from beginning to end.

I am incredibly grateful for this invaluable experience and for my fellow labmates at the University of South Florida. I would like to thank K. Merve Dogan, Emre Yildirim, Dr. Benjamin Gruenwald, S. Burak Sarsilmaz, Jesse Jaramillo, Kevin Wilcher, Stefan Ristevski, Dzung Tran, Dr. Ahmet T. Koru, and Dr. Ehsan Arabi. In addition, I would like to offer my special thanks to K. Merve Dogan for her guidance and enthusiasm to help me every step of the way.

Finally, I would like to thank my parents, my siblings and my friends. Without their love, support and encouragement, I would not have enjoyed or have completed my masters education as successfully. 


\section{Table of Contents}

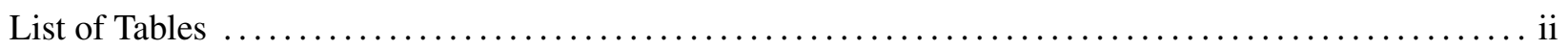

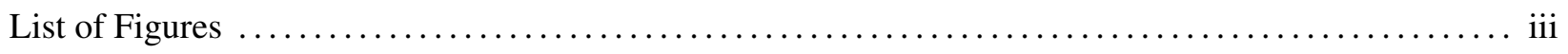

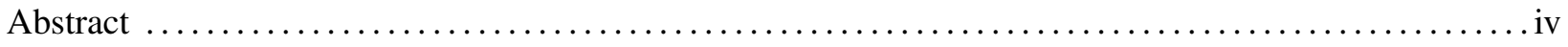

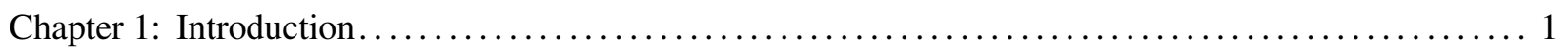

1.1 Model Reference Adaptive Control ..................................... 1

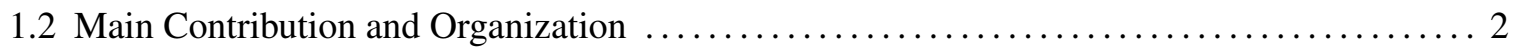

Chapter 2: Application of a Model Reference Adaptive Control Approach

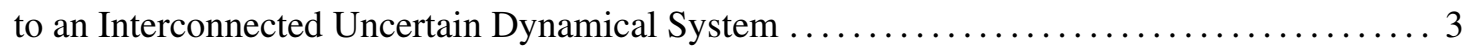

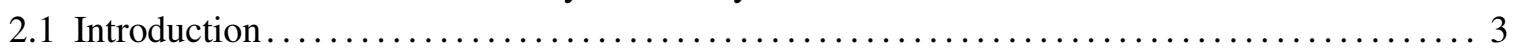

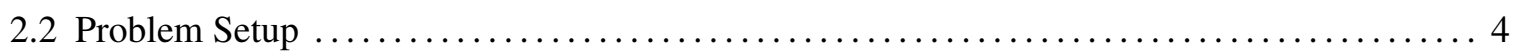

2.3 Experimental Studies on a Benchmark Mechanical System Setup ................... 7

Chapter 3: Concluding Remarks and Future Research $\ldots \ldots \ldots \ldots \ldots \ldots \ldots \ldots \ldots \ldots \ldots \ldots \ldots \ldots$

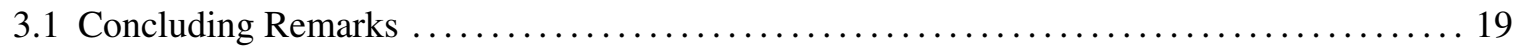

3.2 Future Research ................................................ 19

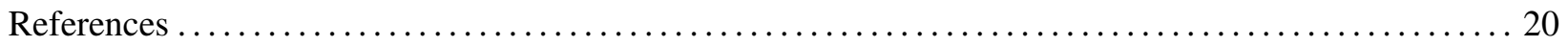

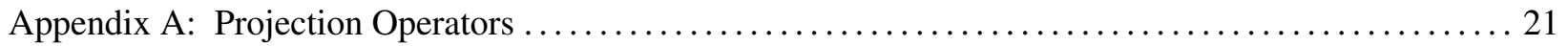




\section{List of Tables}

Table $2.1 \quad$ Notations used in dynamical modeling $\ldots \ldots \ldots \ldots \ldots \ldots \ldots \ldots \ldots \ldots \ldots \ldots \ldots$ 


\section{List of Figures}

Figure $1.1 \quad$ Benchmark mechanical system setup $\ldots \ldots \ldots \ldots \ldots \ldots \ldots \ldots \ldots \ldots \ldots \ldots \ldots \ldots \ldots$

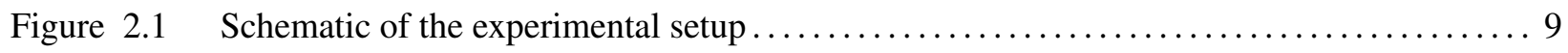

Figure 2.2 Experimental results for the nominal controller (system response and control signals without artificial disturbance and matched uncertainties) $\ldots \ldots \ldots \ldots \ldots \ldots \ldots \ldots \ldots \ldots$

Figure 2.3 Experimental results for the nominal controller (error dependent learning gains and enforced performance bounds without artificial disturbance and matched uncertainties)... 12

Figure 2.4 Experimental results for the nominal controller (system response and control signals

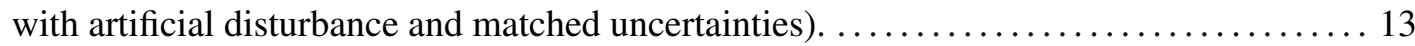

Figure 2.5 Experimental results for the nominal controller (error dependent learning gains and enforced performance bounds with artificial disturbance and matched uncertainties)..... 14

Figure 2.6 Experimental results for proposed set-theoretic adaptive controller (system response and control signals with artificial disturbance and matched uncertainties).

Figure 2.7 Experimental results for proposed set-theoretic adaptive controller (error dependent learning gains and enforced performance bounds for $\varepsilon_{\tilde{z}}=\varepsilon_{e}=1$ with artificial dis-

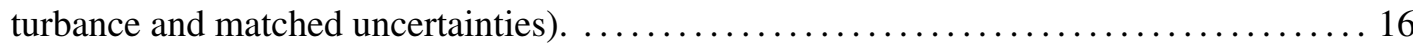

Figure 2.8 Experimental results for proposed set-theoretic adaptive controller (system response and control signals with artificial disturbance and matched uncertainties).

Figure 2.9 Experimental results for proposed set-theoretic adaptive controller (error dependent learning gains and enforced performance bounds for $\varepsilon_{\tilde{z}}=\varepsilon_{e}=0.3$ with artificial disturbance and matched uncertainties). 


\begin{abstract}
The objective of this thesis is to show the experimental validation of recently proposed adaptive control architecture for uncertain dynamical systems. The experimental validation is conducted on a benchmark mechanical system setup composed of two carts, one actuated and one unactuated, physically interconnected through a spring.

Specifically, an approach has been recently proposed to stabilize an overall interconnected system in the presence of unknown physical interconnections as well as system uncertainties in the context of model reference adaptive control. This uncertain dynamical system consists of actuated and unactuated portions physically interconnected to each other. In addition, the previous work enforces performance guarantees individually on both the actuated and unactuated portions of the interconnected system. In particular, a set-theoretic model reference adaptive control approach has been used in conjunction with linear matrix inequalities to enforce these performance guarantees that is restricting the respective system error trajectories of the actuated and unactuated dynamics inside a-priori, user defined compact sets. As stated above, the overarching contribution of this thesis is to present experimental results for the purpose of demonstrating the efficacy of the previously proposed approach on a benchmark mechanical system setup involving an actuated cart coupled with an unactuated cart through a spring in the presence of both unknown friction and unknown uncertainties. It is experimentally observed that utilizing the proposed approach stabilizes and restricts the respective system error trajectories of the interconnected system.
\end{abstract}




\section{Chapter 1: Introduction}

Uncertain dynamical systems consisting of both actuated and unactuated portions physically interconnected to each other behave as underactuated mechanical systems, systems with more degrees of freedom than there are actuators. A wide array of applications include slung-load systems, unactuated fuel slosh dynamics in spacecraft and multibody mobile robots, to name but a few examples. To this end, the authors of [1] have recently studied the uncertain dynamical systems subject to interconnected actuated and unactuated dynamics and proposed an adaptive control architecture with individual performance guarantees enforced on both dynamics. In order to bridge the gap between the theory and practice related to underactuated uncertain dynamical systems, this thesis provides an experimental study on a benchmark mechanical system based on an adaptive control architecture developed by the authors of [1]. For details on the background and literature related to uncertain dynamical systems, we refer the readers to [1].

\subsection{Model Reference Adaptive Control}

Model reference adaptive control is a method consisting of two components, a reference model and a parameter adjustment mechanism. The reference model captures the desired closed-loop dynamical system response and is then compared with the response of the uncertain dynamical system. The parameter adjustment mechanism is then driven by the system error signal resulting from the comparison between the model reference system and the uncertain dynamical system. Through adjusting the controller parameters, the parameter adjustment mechanism is designed (asymptotically or approximately) to drive the trajectories of the uncertain dynamical system to the trajectories of the reference model [2].

This thesis demonstrates the experimental validation of a recently proposed adaptive control architecture in [1] on a benchmark mechanical system setup composed of two carts connected by a spring

to show the efficacy of this architecture in practice, to appear in [3]. It has been shown that through enforcing performance guarantees individually on the actuated and unactuated portions of the interconnected 
dynamical system, the respective system error trajectories are restricted to user-defined compact sets. To display the effectiveness of the proposed adaptive control architecture, experimental plots are shown.

\subsection{Main Contribution and Organization}

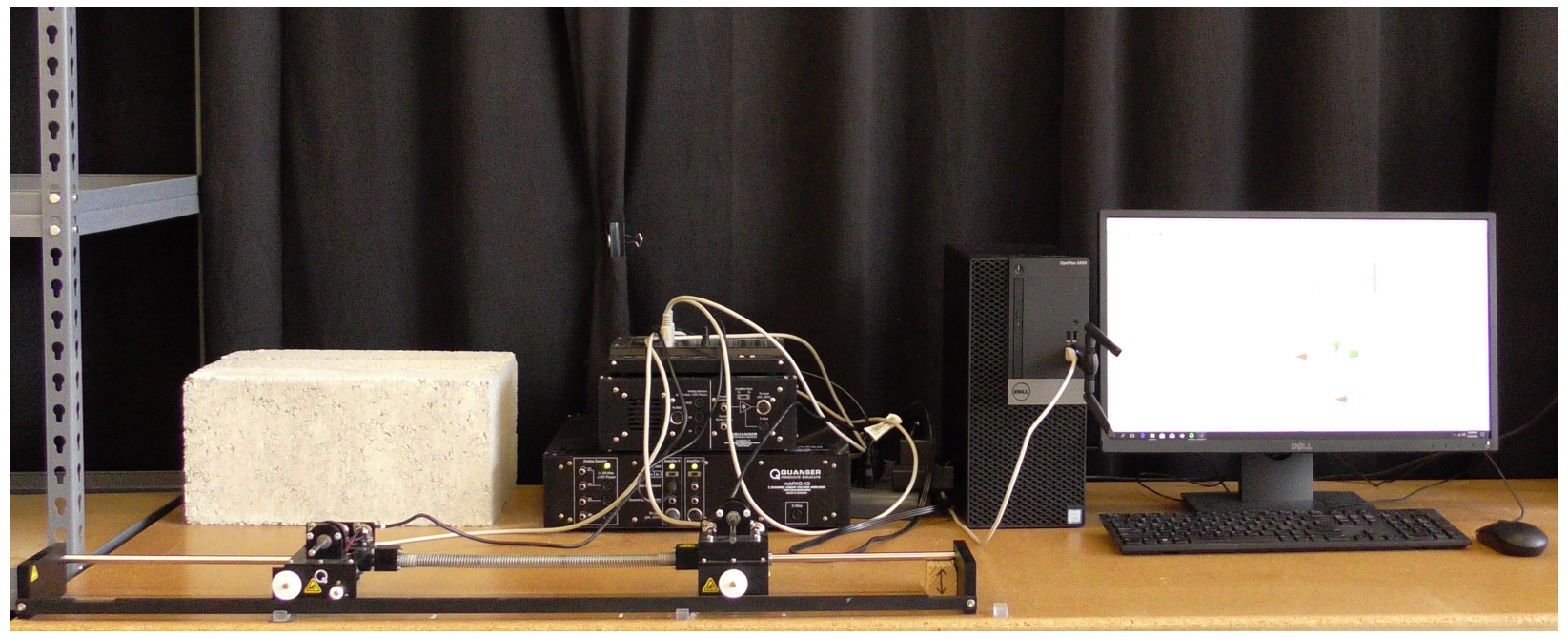

Figure 1.1: Benchmark mechanical system setup

The main contribution of this thesis is to provide an experimental study on a benchmark mechanical system setup. Specifically, this setup is composed of an actuated cart coupled with another unactuated cart through a spring in the presence of unknown frictions and unknown interconnections. Consider the physical system shown in Figure 1.1, where the cart on the left represents the actuated portion that is attached to another cart with a spring and the cart on the right represents the unactuated portion of the dynamical system. We are now ready to state the organization of this thesis. In particular, Chapter 2 first presents an overview of the theory in [1] for completeness. Chapter 2 then presents an experimental study on a benchmark mechanical system setup to show the efficacy of the proposed adaptive control architecture. Finally, concluding remarks and future research directions are presented in Chapter 3. 


\section{Chapter 2: Application of a Model Reference Adaptive Control Approach to an Interconnected Uncertain Dynamical System}

\subsection{Introduction}

We start with outlining the key aspects of the proposed adaptive control architecture in [1], in which the control and performance enforcement of the unactuated dynamics is accomplished through the physical interconnection with the actuated dynamics. Specifically, the previously proposed control is applied to stabilize the overall interconnected system in the presence of unknown physical interconnections as well as uncertainties in both the actuated and unactuated dynamics. The performance guarantees are enforced using a set-theoretic model reference adaptive control approach proposed in [4] such that the respective system error trajectories of the actuated and unactuated dynamics are restricted to stay inside user-defined compact sets. In addition, the previously proposed approach utilizes linear matrix inequalities to verify stability of appropriate control parameters as well as the allowable system uncertainties and unknown physical interconnections. A summary of the conditions and assumptions are given later in this thesis to be selfcontained.

In this thesis, an experimental study is provided on a benchmark mechanical system setup. In particular, this platform is composed of an actuated cart coupled with an unactuated cart through a spring, and is used to experimentally demonstrate the proposed adaptive control approach of [1]. Figure 1.1 shows this platform, where the cart on the left represents the actuated portion physically interconnected with another cart and the cart on the right represents the unactuated portion of the dynamical system. We present an overview of the results in [1], modeling, dynamical equations and the experimental results of this benchmark mechanical system setup later in this thesis.

We are now ready to state the notation used throughout this thesis. Specifically, $\mathbb{R}$ denotes the set of real numbers, $\mathbb{R}^{n}$ denotes the set of $n \times 1$ real column vectors, $\mathbb{R}^{n \times m}$ denotes the set of $n \times m$ real matrices,

$\mathbb{R}_{+}$(resp., $\overline{\mathbb{R}}_{+}$) denotes the set of positive (resp., nonnegative) real numbers, $\mathbb{R}_{+}^{n \times n}$ (resp., $\overline{\mathbb{R}}_{+}^{n \times n}$ ) denotes the set of $n \times n$ positive-definite (resp., nonnegative-definite) real matrices, and " $\triangleq$ " denotes the equality by 
definition throughout this paper. In addition, we use $(\cdot)^{\mathrm{T}}$ for the transpose operator, $(\cdot)^{-1}$ for the inverse operator, $\|\cdot\|_{\mathrm{F}}$ for the Frobenius norm, $\|\cdot\|_{2}$ for the Euclidean norm, and $\lambda_{\max }(A)\left(\operatorname{resp} ., \lambda_{\min }(A)\right)$ for the maximum (resp., minimum) eigenvalue of the matrix $A \in \mathbb{R}^{n \times m}$. We also refer to Appendix A for a necessary definition.

\subsection{Problem Setup}

Consider the class of interconnected uncertain dynamical systems in the form given by

$$
\begin{aligned}
\dot{x}(t) & =A x(t)+B\left[J z(t)+W_{\mathrm{u}}^{\mathrm{T}} x(t)\right], \quad x(0)=x_{0}, \\
\dot{z}(t) & =F z(t)+G\left[u(t)+H x(t)+W_{\mathrm{a}}^{\mathrm{T}} z(t)\right], \quad z(0)=z_{0} .
\end{aligned}
$$

Here, (2.1) represents the unactuated portion of the interconnected system, while (2.2) represents the actuated portion. Specifically, $x(t) \in \mathbb{R}^{n}$ is the measurable state vector of the unactuated dynamics, $z(t) \in \mathbb{R}^{p}$ is the measurable state vector of the actuated dynamics, $u(t) \in \mathbb{R}^{q}$ is the control input. $A \in \mathbb{R}^{n \times n}$ is a known system matrix, $B \in \mathbb{R}^{n \times m}$ is a known input matrix. Note that for the unactuated dynamics it is assumed the pair $(A, B)$ is controllable. In (2), $F \in \mathbb{R}^{p \times p}$ is a known system matrix and $G \in \mathbb{R}^{p \times q}$ is a known input matrix. Note also that for the actuated dynamics it is assumed the pair $(F, G)$ is controllable. In both (2.1) and (2.2), $W_{u} \in \mathbb{R}^{n \times m}$ is an unknown weight matrix representing uncertainty in the unactuated dynamics and $W_{\mathrm{a}} \in \mathbb{R}^{p \times q}$ is an unknown weight matrix representing uncertainty in the actuated dynamics. In addition, $J \in \mathbb{R}^{m \times p}$ represents the effect on the unactuated dynamics from the unknown physical interconnection with the actuated dynamics and $H \in \mathbb{R}^{q \times n}$ represents the effect on the actuated dynamics from the unknown physical interconnection with the unactuated dynamics. Here, we consider these unknown physical interconnections to be parameterized as

$$
\begin{aligned}
H & =H_{0}+H_{\Delta}, \\
J & =J_{0}+J_{\Delta} .
\end{aligned}
$$

In (2.3) and (2.4), $H_{0} \in \mathbb{R}^{q \times n}$ and $J_{0} \in \mathbb{R}^{m \times p}$ are known parts of the physical interconnection while $H_{\Delta} \in \mathbb{R}^{q \times n}$ and $J_{\Delta} \in \mathbb{R}^{m \times p}$ are their unknown parts. Note that the objective of [1] is to design a control signal for the actuated dynamics given by (2.2) such that the trajectories of the actuated dynamics follow the trajectories of 
a desired reference model, the trajectories of the unactuated dynamics given by (2.1) follow the trajectories of a desired reference model, and the respective system error trajectories of the actuated and unactuated dynamics are restricted to a-priori, user-defined compact sets enforcing performance guarantees. To begin, the following necessary definition from [4] (also used in [1]) is provided.

Definition 1. Let $\|y\|_{M}=\sqrt{y^{T} M y}$ be a weighted Euclidean norm with a real column vector $y \in \mathbb{R}^{s}$ and a matrix $M \in \mathbb{R}_{+}^{s \times s}$. Then one can define $\phi\left(\|y\|_{M}\right), \phi: \mathbb{R}^{s} \rightarrow \mathbb{R}$, to be a restricted potential function (barrier Lyapunov function) defined on the set $\mathcal{D}_{\varepsilon} \triangleq y:\|y\|_{M} \in[0, \varepsilon)$ with a-priori, user defined constant $\varepsilon \in \mathbb{R}_{+}$. In addition, if the following statements hold:

i) If $\|y\|_{M}=0$, then $\phi\left(\|y\|_{M}\right)=0$.

ii) If $y \in \mathcal{D}_{\varepsilon}$ and $\|y\|_{M} \neq 0$, then $\phi\left(\|y\|_{M}\right)>0$.

iii) If $\|y\|_{M} \rightarrow \varepsilon$, then $\phi\left(\|y\|_{M}\right) \rightarrow \infty$.

iv) $\phi\left(\|y\|_{M}\right)$ is continuously differentiable on $\mathcal{D}_{\varepsilon}$.

v) If $y \in \mathcal{D}_{\varepsilon}$, then $\phi_{d}\left(\|y\|_{M}\right)>0$, where $\phi_{d}\left(\|y\|_{M}\right) \triangleq \frac{d \phi\left(\|y\|_{M}\right)}{d\|y\|_{M}^{2}}$.

vi) If $y \in \mathcal{D}_{\varepsilon}$, then $2 \phi_{d}\left(\|y\|_{M}\right)\|y\|_{M}^{2}-\phi\left(\|y\|_{M}\right)>0$.

Now, we consider the adaptive control architecture to control the actuated dynamics subject to both system uncertainties and unknown physical interconnections with unactuated dynamics,

$$
u(t)=-K_{1} z(t)+K_{2} u_{1}(t)-\left(H_{0}+\hat{H}_{\Delta}(t)\right) x(t)-\hat{W}_{\mathrm{a}}^{\mathrm{T}}(t) z(t) .
$$

Here, $K_{1} \in \mathbb{R}^{q \times p}$ is designed such that $F_{\mathrm{r}} \triangleq F-G K_{1}$ is Hurwitz ${ }^{1}, K_{2} \in \mathbb{R}^{q \times m}$ is designed such that $-J_{0}\left(F-G K_{1}\right)^{-1} G K_{2}=I$, and $u_{1}(t) \in \mathbb{R}^{m}$ is an additional control signal to be designed by taking into account the unactuated dynamics. In (2.5), $\hat{H}_{\Delta}(t) \in \mathbb{R}^{q \times n}$ and $\hat{W}_{\mathrm{a}} \in \mathbb{R}^{p \times q}$ are the estimates of unknown parts of $H_{\Delta}$ and $W_{\mathrm{a}}$ satisfying the respective projection operator based weight update laws

$$
\begin{aligned}
& \dot{\hat{H}}_{\Delta}(t)=\alpha \operatorname{Proj}_{\mathrm{m}}\left[\hat{H}_{\Delta}(t), \phi_{\mathrm{d}}\left(\|\tilde{z}(t)\|_{S}\right) G^{\mathrm{T}} S \tilde{z}(t) x^{\mathrm{T}}(t)\right], \quad \hat{H}_{\Delta}(0)=\hat{H}_{\Delta 0}, \\
& \dot{\hat{W}}_{\mathrm{a}}(t)=\gamma_{\mathrm{a}} \operatorname{Proj}_{\mathrm{m}}\left[\hat{W}_{\mathrm{a}}(t), \phi_{\mathrm{d}}\left(\|\tilde{z}(t)\|_{S}\right) z(t) \tilde{z}^{\mathrm{T}}(t) S G\right], \quad \hat{W}_{\mathrm{a}}(0)=\hat{W}_{\mathrm{a} 0} .
\end{aligned}
$$

In (2.6) and (2.7), $\alpha \in \mathbb{R}_{+}$and $\gamma_{\mathrm{a}} \in \mathbb{R}_{+}$are the learning rates, $\phi_{\mathrm{d}}\left(\|\tilde{z}(t)\|_{S}\right)$ is an error dependent learning gain, and $\tilde{z}(t) \triangleq z(t)-z_{\mathrm{r}}(t)$ is the system error state vector of the actuated dynamics with $z_{\mathrm{r}}(t) \in \mathbb{R}^{p}$

\footnotetext{
${ }^{1}$ There exists $S \in \mathbb{R}_{+}^{p \times p}$ such that $0=F_{r}^{\mathrm{T}} S+S F_{r}+I$.
} 
being the reference state vector satisfying the reference model dynamics given by

$$
\dot{z}_{\mathrm{r}}(t)=F_{\mathrm{r}} z_{\mathrm{r}}(t)+G_{\mathrm{r}} u_{1}(t), \quad z_{\mathrm{r}}(0)=z_{\mathrm{r} 0}
$$

Here, $G_{\mathrm{r}}=G K_{2} \in \mathbb{R}^{p \times m}$ is the reference model input matrix. In addition, the remaining control signal $u_{1}(t)$ is designed to allow command following of the unactuated dynamics. This signal is applied to the actuated dynamics to control the unactuated dynamics through the physical interconnection of the two dynamics and is given by

$$
u_{1}(t)=-L_{1} x(t)+L_{2} c(t)-\hat{J}_{\Delta}(t) z(t)+\hat{W}_{\mathrm{u}}^{\mathrm{T}}(t) x(t)
$$

where $L_{1} \in \mathbb{R}^{m \times n}$ is designed such that $A_{\mathrm{r}} \triangleq A-B L_{1}$ is Hurwitz ${ }^{2}, L_{2} \in \mathbb{R}^{m \times m}$ is a feedforward gain, and $c(t) \in \mathbb{R}^{m}$ is a given uniformly continuous bounded command. In (2.9), $\hat{J}_{\Delta}(t) \in \mathbb{R}^{m \times p}$ and $\hat{W}_{\mathrm{u}}(t) \in \mathbb{R}^{n \times m}$ are the estimates of unknown parts $J_{\Delta}$ and $W_{\mathrm{u}}$ satisfying the respective projection operator based weight update laws given by

$$
\begin{aligned}
\dot{\hat{J}}_{\Delta}(t)=\beta \operatorname{Proj}_{\mathrm{m}}\left[\hat{J}_{\Delta}(t), \phi_{\mathrm{d}}\left(\|e(t)\|_{P}\right) B^{\mathrm{T}} P e(t) z^{\mathrm{T}}(t)\right], & \hat{J}_{\Delta}(0)=\hat{J}_{\Delta 0}, \\
\dot{\hat{W}}_{\mathrm{u}}(t)=\gamma_{\mathrm{u}} \operatorname{Proj}_{\mathrm{m}}\left[\hat{W}_{\mathrm{u}}(t), \phi_{\mathrm{d}}\left(\|e(t)\|_{P}\right) x(t) e^{\mathrm{T}}(t) P B\right], & \hat{W}_{\mathrm{u}}(0)=\hat{W}_{\mathrm{u} 0} .
\end{aligned}
$$

In (2.10) and (2.11), $\beta \in \mathbb{R}_{+}$and $\gamma_{\mathrm{u}} \in \mathbb{R}_{+}$are the learning rates and $e(t) \triangleq x(t)-x_{\mathrm{r}}(t)$ is the system error state vector of the unactuated dynamics with $x_{\mathrm{r}}(t) \in \mathbb{R}^{n}$ being the reference state vector satisfying the reference model dynamics given by

$$
\dot{x}_{\mathrm{r}}(t)=A_{\mathrm{r}} x_{\mathrm{r}}(t)+B_{\mathrm{r}} c(t)+B\left[J_{0} z(t)-u_{1}(t)\right], \quad x_{\mathrm{r}}(0)=x_{\mathrm{r} 0} .
$$

Here, $B_{\mathrm{r}}=B L_{2} \in \mathbb{R}^{n \times m}$ is the reference model input matrix. Now, the stability and performance guarantees for the proposed adaptive control architecture is overviewed in [1]. The resulting actuated system error dynamics are given by

$$
\dot{\tilde{z}}(t)=F_{\mathrm{r}} \tilde{z}(t)-G \tilde{H}_{\Delta}(t) x(t)-G \tilde{W}_{\mathrm{a}}^{\mathrm{T}}(t) z(t), \quad \tilde{z}(0)=\tilde{z}_{0} .
$$

\footnotetext{
${ }^{2}$ There exists $P \in \mathbb{R}_{+}^{n \times n}$ such that $0=A_{\mathrm{r}}^{\mathrm{T}} P+P A_{\mathrm{r}}+I$.
} 
In (2.13), $\tilde{H}_{\Delta}(t) \triangleq \hat{H}_{\Delta}(t)-H_{\Delta} \in \mathbb{R}^{q \times n}$ and $\tilde{W}_{\mathrm{a}}(t) \triangleq \hat{W}_{\mathrm{a}}(t)-W_{\mathrm{a}} \in \mathbb{R}^{p \times q}$. The resulting unactuated system error dynamics are given by

$$
\dot{e}(t)=A_{\mathrm{r}} e(t)-B \tilde{J}_{\Delta}(t) z(t)-B \tilde{W}_{\mathrm{u}}^{\mathrm{T}}(t) x(t), \quad e(0)=e_{0} .
$$

In (2.14), $\tilde{J}_{\Delta}(t) \triangleq \hat{J}_{\Delta}(t)-J_{\Delta} \in \mathbb{R}^{q \times n}$ and $\tilde{W}_{\mathrm{u}}(t) \triangleq \hat{W}_{\mathrm{u}}(t)-W_{\mathrm{u}} \in \mathbb{R}^{n \times m}$ are weight estimation errors. The following assumption is now needed.

Assumption 1. The matrix

$$
\mathcal{A}\left(\hat{J}_{\Delta}(t), \hat{W}_{\mathrm{u}}(t)\right)=\left[\begin{array}{cc}
A+B \hat{W}_{\mathrm{u}}^{\mathrm{T}}(t) & B\left(J_{0}+\hat{J}_{\Delta}(t)\right) \\
-G_{\mathrm{r}}\left(L_{1}+\hat{W}_{\mathrm{u}}^{\mathrm{T}}(t)\right) & F_{\mathrm{r}}-G_{\mathrm{r}} \hat{J}_{\Delta}(t)
\end{array}\right]
$$

is quadratically stable ${ }^{3}$.

Considering the proposed model reference adaptive control approach, one can show that if $\left\|\tilde{z}_{0}\right\|_{S}<$ $\varepsilon_{\tilde{\mathrm{Z}}}$ and $\left\|e_{0}\right\|_{\mathrm{P}}<\varepsilon_{\mathrm{e}}$, then under Assumption 1, the solution $\left(\tilde{z}(t), e(t), \tilde{H}_{\Delta}(t), \tilde{W}_{\mathrm{a}}(t), \tilde{J}_{\Delta}(t), \tilde{W}_{\mathrm{u}}(t)\right)$ of the closedloop interconnected dynamical system is bounded, $\lim _{t \rightarrow \infty} \tilde{z}(t)=0$, and $\lim _{t \rightarrow \infty} e(t)=0$ [1]. Note that under Assumption 1, the upper bound for $\left\|\tilde{x}_{\mathrm{r}}(t)\right\|_{2}$ can be written as

$$
\left\|\tilde{x}_{\mathrm{r}}(t)\right\|_{2} \leq \sqrt{\frac{\lambda_{\max }(\mathcal{P})}{\lambda_{\min }(\mathcal{P})}} \Theta^{*}
$$

where $\Theta^{*}$ has the following form

$$
\Theta^{*} \triangleq 2 \rho^{-1}\|\mathcal{P B}\|_{\mathrm{F}}\left[\psi^{*}\left(\frac{\varepsilon_{e}}{\sqrt{\lambda_{\min }(P)}}+x_{\mathrm{r}}^{*}\right)+\phi^{*}\left(\frac{\varepsilon_{\tilde{\mathrm{z}}}}{\sqrt{\lambda_{\min }(S)}}\right)+\left\|L_{2}\right\|_{\mathrm{F}} c^{*}\right]
$$

In (2.17), $\left\|x_{\mathrm{r}}^{\mathrm{id}}(t)\right\|_{2} \leq x_{\mathrm{r}}^{*}$, where $x_{\mathrm{r}}^{\mathrm{id}}(t) \in \mathbb{R}^{n}$ is the ideal reference state satisfying $\dot{x}_{\mathrm{r}}^{\mathrm{id}}(t)=A_{\mathrm{r}} x_{\mathrm{r}}^{\mathrm{id}}(t)+B_{\mathrm{r}} c(t)$, where $\|c(t)\|_{2} \leq c^{*}$. In addition, $\mathcal{B}=\left[B^{\mathrm{T}},-G_{\mathrm{r}}^{\mathrm{T}}\right]^{\mathrm{T}},\left\|L_{1}+\hat{W}_{\mathrm{u}}^{\mathrm{T}}(t)\right\|_{\mathrm{F}} \leq \psi^{*}$ and $\left\|J_{0}+\hat{J}_{\Delta}(t)\right\|_{\mathrm{F}} \leq \phi^{*}$.

\subsection{Experimental Studies on a Benchmark Mechanical System Setup}

A schematic of the benchmark mechanical system setup in Figure 1.1 is now shown in Figure 2.1. This setup consists of parts produced by Quanser and assembled in our research laboratory [5]. This setup

\footnotetext{
${ }^{3}$ Since $\mathcal{A}\left(\hat{J}_{\Delta}(t), \hat{W}_{\mathrm{u}}(t)\right)$ is quadratically stable, it implies that $\mathcal{A}^{\mathrm{T}}(\cdot) \mathcal{P}+\mathcal{P} \mathcal{A}(\cdot)<0$, where $\mathcal{P} \in \mathbb{R}_{+}$.
} 
involves a cart coupled with another cart through a spring in the presence of both unknown friction and unknown interconnections. This setup is also comprised of two carts in which the first cart is driven by a rack and pinion mechanism using a 6 Volt DC motor, ensuring consistent and continuous traction. The carts slide along a steel shaft using linear bearings moving only in $\mathrm{x}$ direction. The linear voltage controlled power amplifier VoltPAQ-X1 is used to drive this experiment. The positions of the two carts are measured using a sensor coupled to the rack via an additional pinion. The proposed control approach discussed in Section 2.2 is implemented on the computer and run on MATLAB/Simulink by using Quanser Quarc Real Time Windows Target (Win64). The data transmission between the computer and the drivers is carried out with digital to analog converter Q8-USB data acquisition board. To summarize, the first cart represents the actuated portion and the second cart represents the unactuated portion of the interconnected system.

Mathematically speaking, we first begin by presenting the equations of motion of this experimental setup. The dynamics of the considered benchmark mechanical system setup satisfies

$$
\begin{aligned}
& {\left[\begin{array}{c}
\dot{z}(t) \\
\ddot{z}(t)
\end{array}\right]=\underbrace{\left[\begin{array}{cc}
0 & 1 \\
-\frac{k_{0}}{m_{z}} & -\frac{b_{0} R_{m} r_{m p}^{2}-\eta_{g} K_{g}^{2} K_{t} K_{m}}{m_{z} R_{m} r_{m p}^{2}}
\end{array}\right]}_{F}\left[\begin{array}{c}
z(t) \\
\dot{z}(t)
\end{array}\right]+\underbrace{\left[\begin{array}{c}
0 \\
\frac{\eta_{g} K_{g} K_{t} \eta_{m}}{m_{z} R_{m} r_{m p}}
\end{array}\right]}_{G}(u(t)+\underbrace{\left[\begin{array}{cc}
\frac{k_{0} R_{m} r_{m p}}{\eta_{g} K_{g} K_{t} \eta_{m}} & 0
\end{array}\right]}_{H_{0}}\left[\begin{array}{c}
x \\
\dot{x}
\end{array}\right]}
\end{aligned}
$$

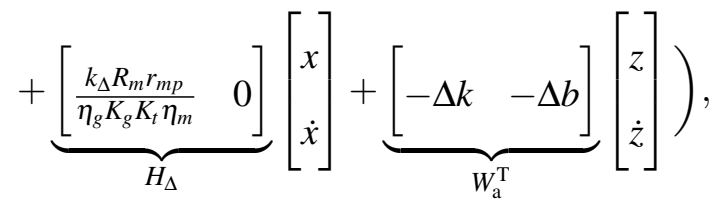

$$
\begin{aligned}
& {\left[\begin{array}{c}
\dot{x}(t) \\
\ddot{x}(t)
\end{array}\right]=\underbrace{\left[\begin{array}{cc}
0 & 1 \\
-\frac{k_{0}}{m_{x}} & -\frac{b_{0}}{m_{x}}
\end{array}\right]}_{A}\left[\begin{array}{c}
x(t) \\
\dot{x}(t)
\end{array}\right]+\underbrace{\left[\begin{array}{c}
0 \\
\frac{1}{m_{x}}
\end{array}\right]}_{B}(\underbrace{\left[\begin{array}{cc}
k_{0} & 0
\end{array}\right]}_{J_{0}}\left[\begin{array}{c}
z \\
\dot{z}
\end{array}\right]+\underbrace{\left[\begin{array}{cc}
\Delta k & 0
\end{array}\right]}_{J_{\Delta}}\left[\begin{array}{c}
z \\
\dot{z}
\end{array}\right]+\underbrace{\left[\begin{array}{cc}
-\Delta k & -\Delta b
\end{array}\right]}_{W_{\mathrm{u}}^{\mathrm{T}}}\left[\begin{array}{c}
x \\
\dot{x}
\end{array}\right]) .}
\end{aligned}
$$

In (2.18) and (2.19), $\Delta k$ denotes the unknown part of the spring coefficient and $\Delta b$ denotes the unknown part of the friction, where all other known parameters are given in Table 2.1. The variables $z(t)$ and $x(t)$ denote the position of cart 1 and cart 2, respectively. The variable $u(t)$ denotes the voltage, which is applied to the cart as a control input. 
Table 2.1: Notations used in dynamical modeling

\begin{tabular}{|c|c|c|}
\hline$\tau_{m}$ & Motor Efficiency & 1 \\
\hline$R_{m}$ & Motor Armature Resistance & 2.6 \\
\hline$\tau_{g}$ & Planetary Gearbox Efficiency & 1 \\
\hline$g$ & Gravitational Constant on Earth & $9.79 \mathrm{~m} / \mathrm{s}^{2}$ \\
\hline$K_{g}$ & Planetary Gearbox Gear Ratio & 3.71 \\
\hline$M\left(m_{x}=m_{z}\right)$ & Mass of Cart & $0.507 \mathrm{~kg}$ \\
\hline$K_{t}$ & Motor Current Torque Constant & $7.68 \times 10^{-3} \mathrm{Nm} / \mathrm{A}$ \\
\hline$r_{m p}$ & Motor Pinion Radius & $6.35 \times 10^{-3} \mathrm{~m}$ \\
\hline$K_{m}$ & Motor Back-emf Constant & $7.68 \times 10^{-3} \mathrm{~V} /(\mathrm{rad} / \mathrm{s})$ \\
\hline$J_{m}$ & Motor Moment of Inertia & $3.9 \times 10^{-7} \mathrm{kgm}$ \\
\hline$k_{0}$ & Spring Constant & $160 \mathrm{~N} / \mathrm{m}$ \\
\hline$b_{0}$ & Equivalent Viscous Damping Coefficient at the cart & $1.1 \mathrm{Nms} / \mathrm{rad}$ \\
\hline
\end{tabular}

Here, we consider the benchmark mechanical system setup involving an actuated cart coupled with an unactuated cart through a spring in the presence of unknown frictions as well as unknown physical

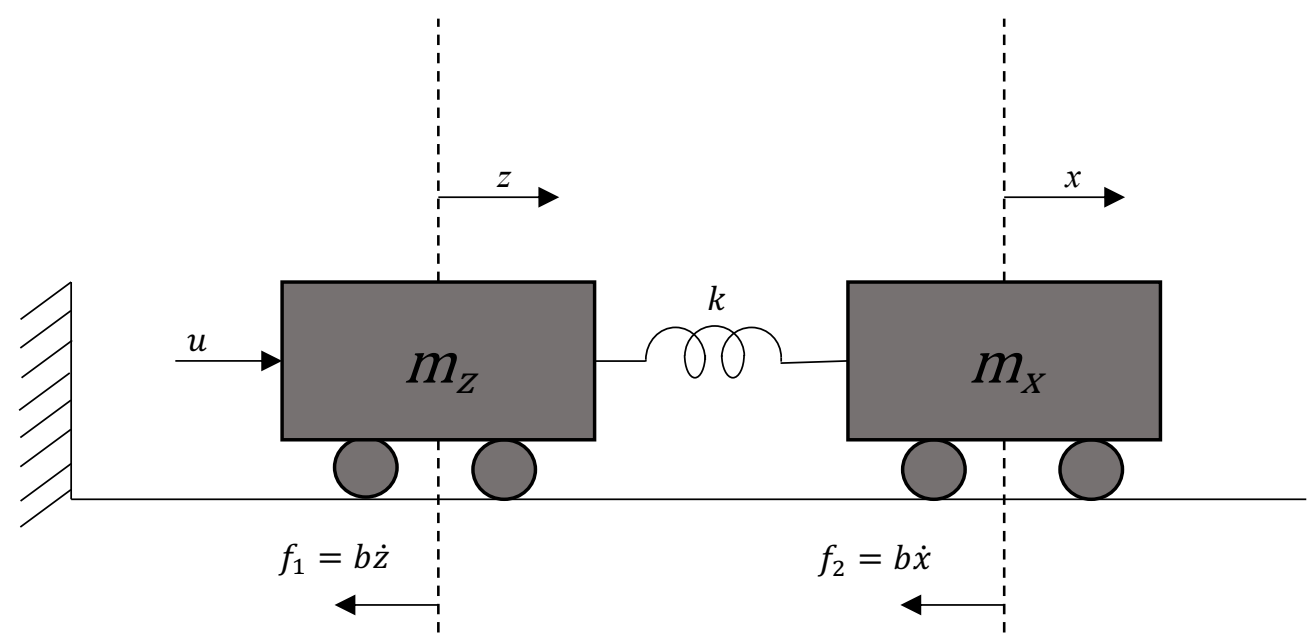

Figure 2.1: Schematic of the experimental setup 
interconnections. In addition, we artificially add disturbance and matched uncertainties (i.e. $\delta+W^{\mathrm{T}} \sigma$, where $\delta=0.1, W^{\mathrm{T}}=[0.5,0.5]$, and $\left.\sigma=[z, 1]\right)$. We first present the nominal control law response.

The nominal control law gain matrices $K_{1}$ and $L_{1}$ for the actuated and unactuated cart, respectively, are obtained through a linear quadratic regulator designed with weightening matrices $Q_{\mathrm{a}}=\operatorname{diag}[8000,400]$ to penalize the states of the actuated cart and $R_{\mathrm{a}}=0.10$ to penalize the control input, $\mathrm{u}(\mathrm{t}) . K_{1}$ is calculated to be $K_{1}=[204.86,60.65]$, allowing $F_{\mathrm{r}}$ to be designed such that it is Hurwitz for the actuated cart. The feedfoward gain $K_{2}$ is designed such that $-J_{0}\left(F-G K_{1}\right)^{-1} G K_{2}=1$, where $J_{0}=\left[k_{0}, 0\right]$ which gives a value of $K_{2}=1.86$. The weighting matrices for the unactuated cart is selected to be $Q_{\mathrm{u}}=\operatorname{diag}[600,400]$ to penalize the states and $R_{\mathrm{a}}=0.10$ to penalize the control input $u_{1}(t)$. For the unactuated cart, $L_{1}=[17.76,62.34]$ and is used to design a Hurwitz reference model matrix $A_{\mathrm{r}}$. The gain $L_{2}$ is calculated as $L_{2}=-\left(C\left(A-B L_{1}\right)^{-1}\right)^{-1}=$ 177.76 where $C=[1,0]$ using a pre-filter such that a desired position for the unactuated cart is followed.

Using the rectangular projection operator, the bounds are set to be $[-1.5,1.5]$. For both the nominal and proposed control architectures, a command $c(t)$ is set such that it changes smoothly from $0.05 m$ to $-0.05 m$, learning rates $\gamma_{\mathrm{a}}, \gamma_{\mathrm{u}}, \alpha, \beta$ are set as $\gamma_{\mathrm{a}}=1, \gamma_{\mathrm{u}}=1, \alpha=1$ and $\beta=1$. Focusing on the error dependent learning gains $\phi_{\mathrm{d}}\left(\|\tilde{z}(t)\|_{S}\right)$ and $\phi_{\mathrm{d}}\left(\|e(t)\|_{P}\right)$ to enforce the performance bounds are set as $\varepsilon_{\tilde{z}}=\varepsilon_{e}=0.3$. Figures 2.2 and 2.3 show the nominal control performance, control input, error dependent learning gains, and enforced performance bounds of the actuated and unactuated cart. In addition, Figures 2.4 and 2.5 show the nominal control performance of the actuated and unactuated carts with added artificial disturbances and matched uncertainties, as well as control input, error dependent learning gains and enforced performance bounds.

We now show the proposed set-theoretic adaptive control architecture response studied in Section 2.2. In particular, Figures 2.6 and 2.7 respectively show the experimental results of the proposed set-theoretic adaptive controller performance for interconnected actuated and unactuated carts with added artificial disturbance and matched uncertainties as well as the control signals, error dependent learning gains and the enforced performance bounds. The performance bound is first set to $\varepsilon_{\tilde{z}}=\varepsilon_{e}=1$ keeping the error dependent learning gains $\phi_{d}\left(\|\tilde{z}\|_{S}\right)$ and $\phi_{d}\left(\|e\|_{P}\right)$ small, as shown in Figure 2.7. Figures 2.8 and 2.9 show the experimental results of the proposed set-theoretic adaptive controller when enforcing the performance bounds at $\varepsilon_{\tilde{z}}=\varepsilon_{e}=0.3$. Validating the illustrative numerical example in [1], once the performance bounds were decreased the need for the error dependent learning gains is more evident. Comparing the error dependent learning rates in Figures 2.7 and 2.9 shows a significant increase in magnitude. 

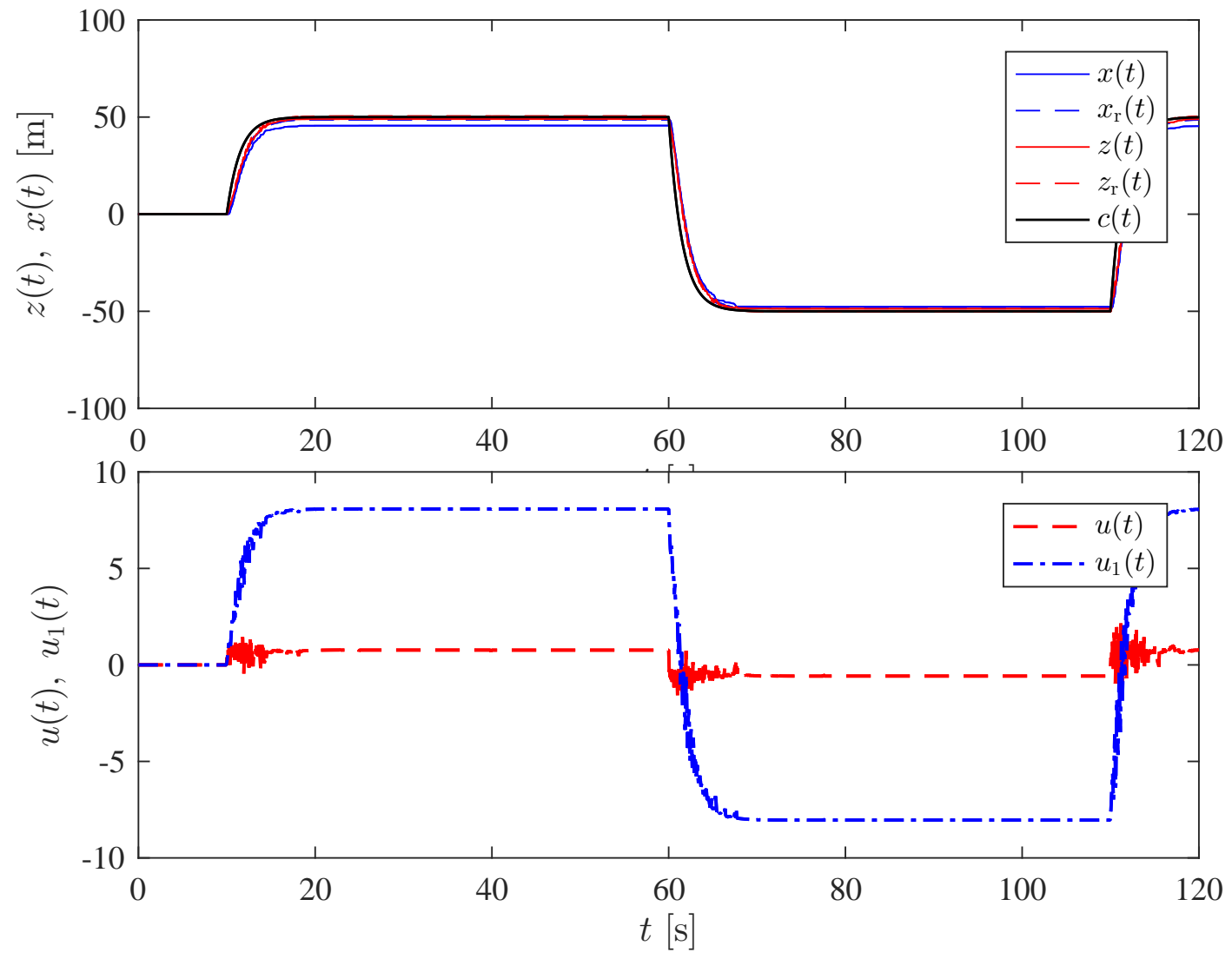

Figure 2.2: Experimental results for the nominal controller (system response and control signals without artificial disturbance and matched uncertainties). 

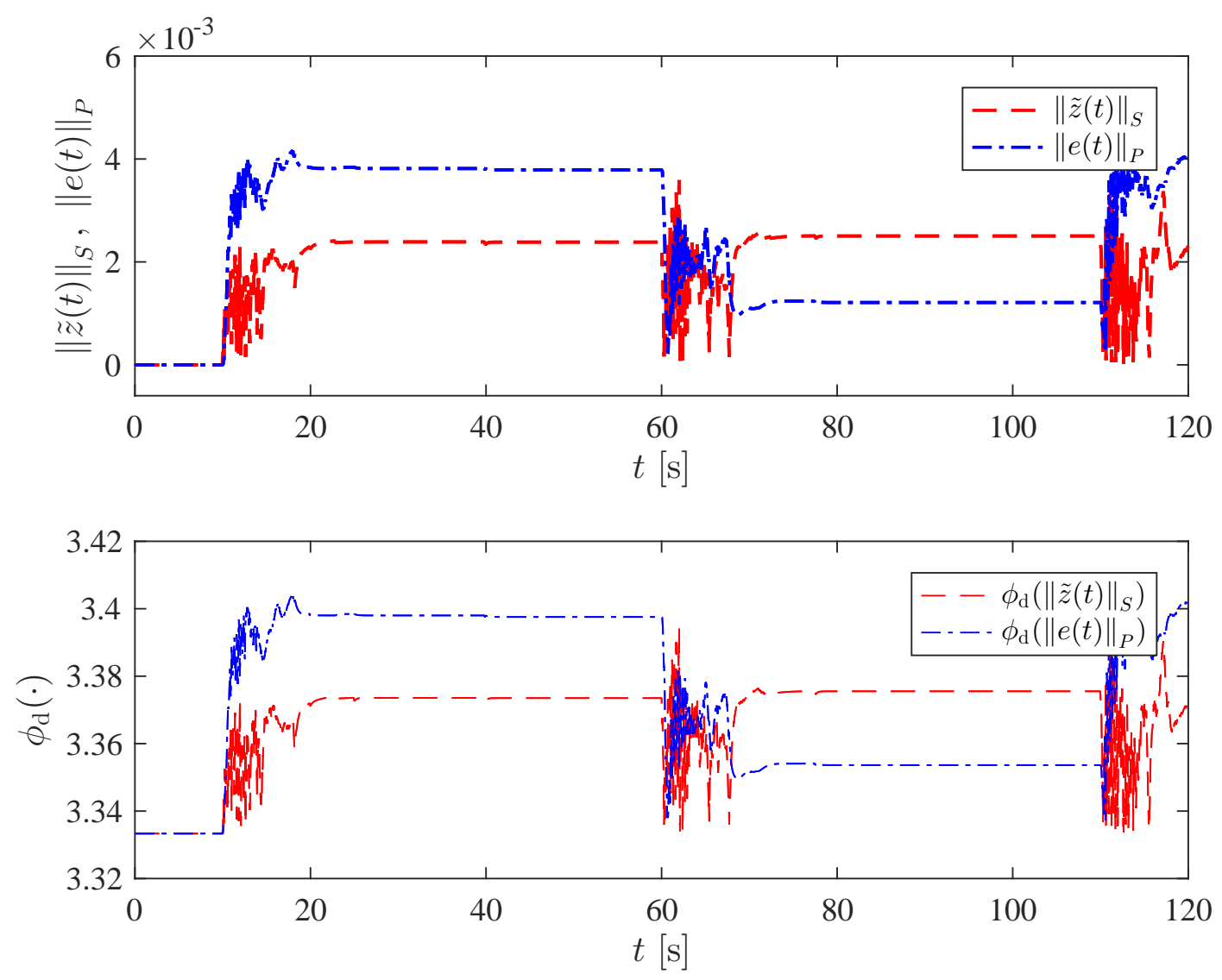

Figure 2.3: Experimental results for the nominal controller (error dependent learning gains and enforced performance bounds without artificial disturbance and matched uncertainties). 

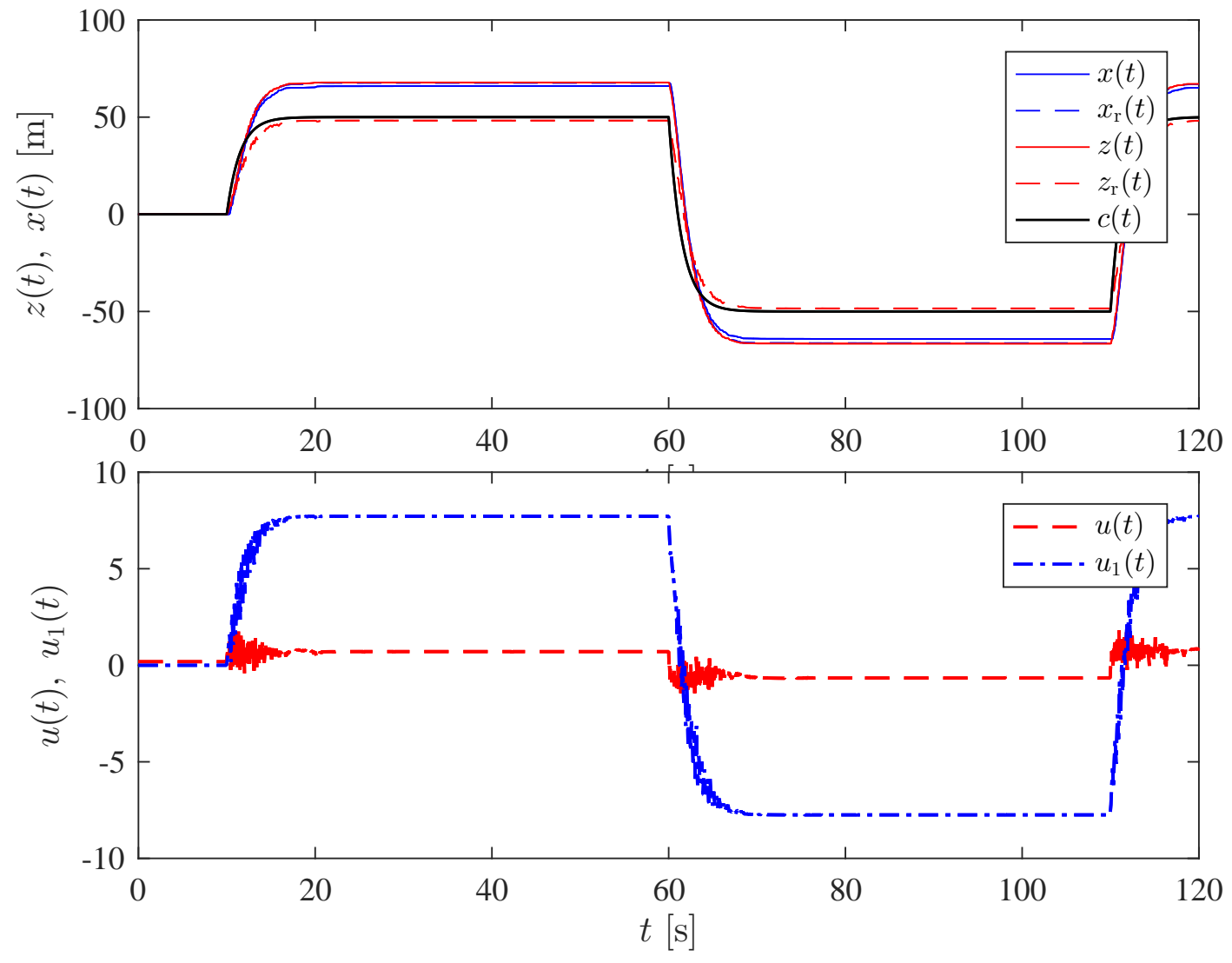

Figure 2.4: Experimental results for the nominal controller (system response and control signals with artificial disturbance and matched uncertainties). 

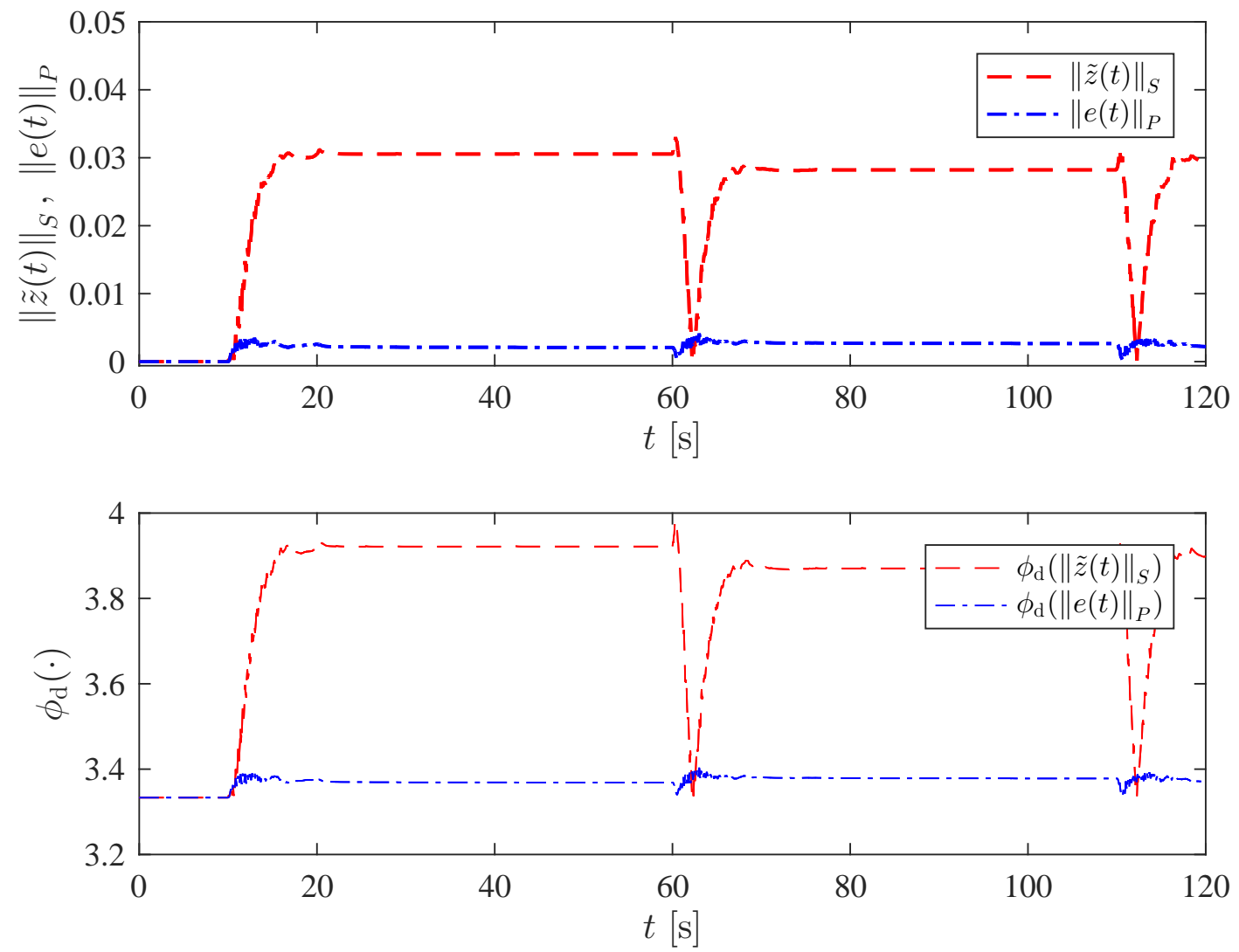

Figure 2.5: Experimental results for the nominal controller (error dependent learning gains and enforced performance bounds with artificial disturbance and matched uncertainties). 

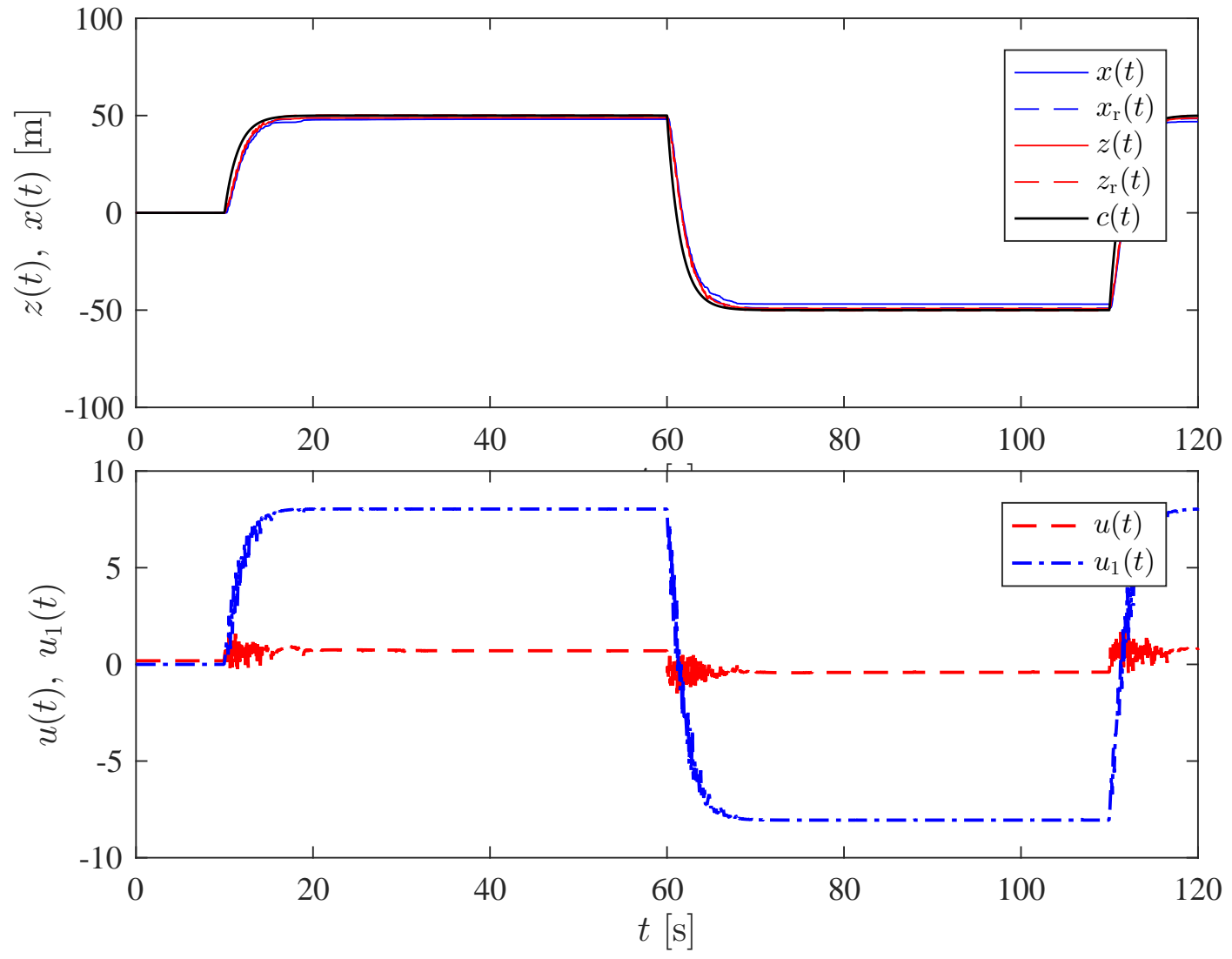

Figure 2.6: Experimental results for proposed set-theoretic adaptive controller (system response and control signals with artificial disturbance and matched uncertainties). 

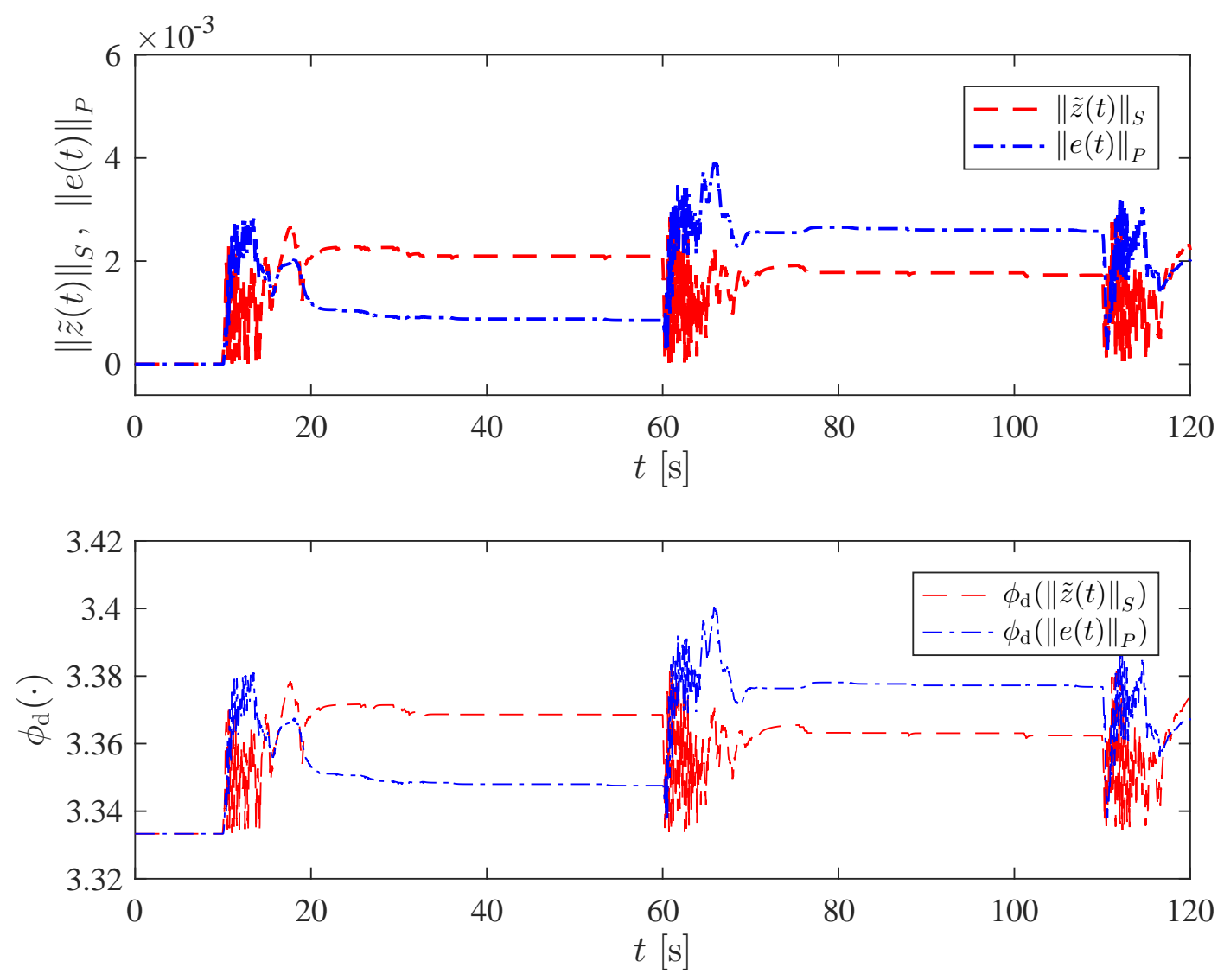

Figure 2.7: Experimental results for proposed set-theoretic adaptive controller (error dependent learning gains and enforced performance bounds for $\varepsilon_{\tilde{z}}=\varepsilon_{e}=1$ with artificial disturbance and matched uncertainties). 

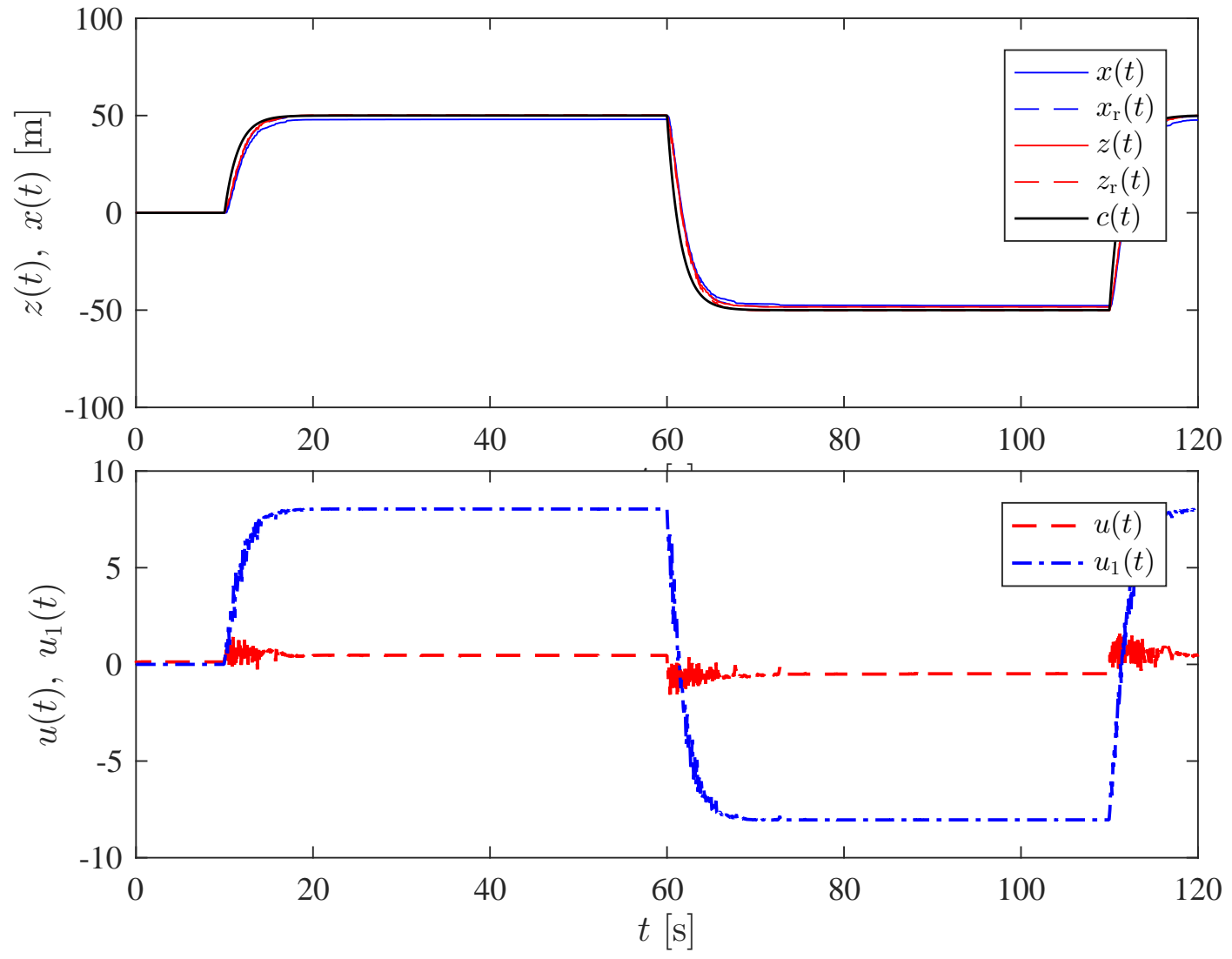

Figure 2.8: Experimental results for proposed set-theoretic adaptive controller (system response and control signals with artificial disturbance and matched uncertainties). 

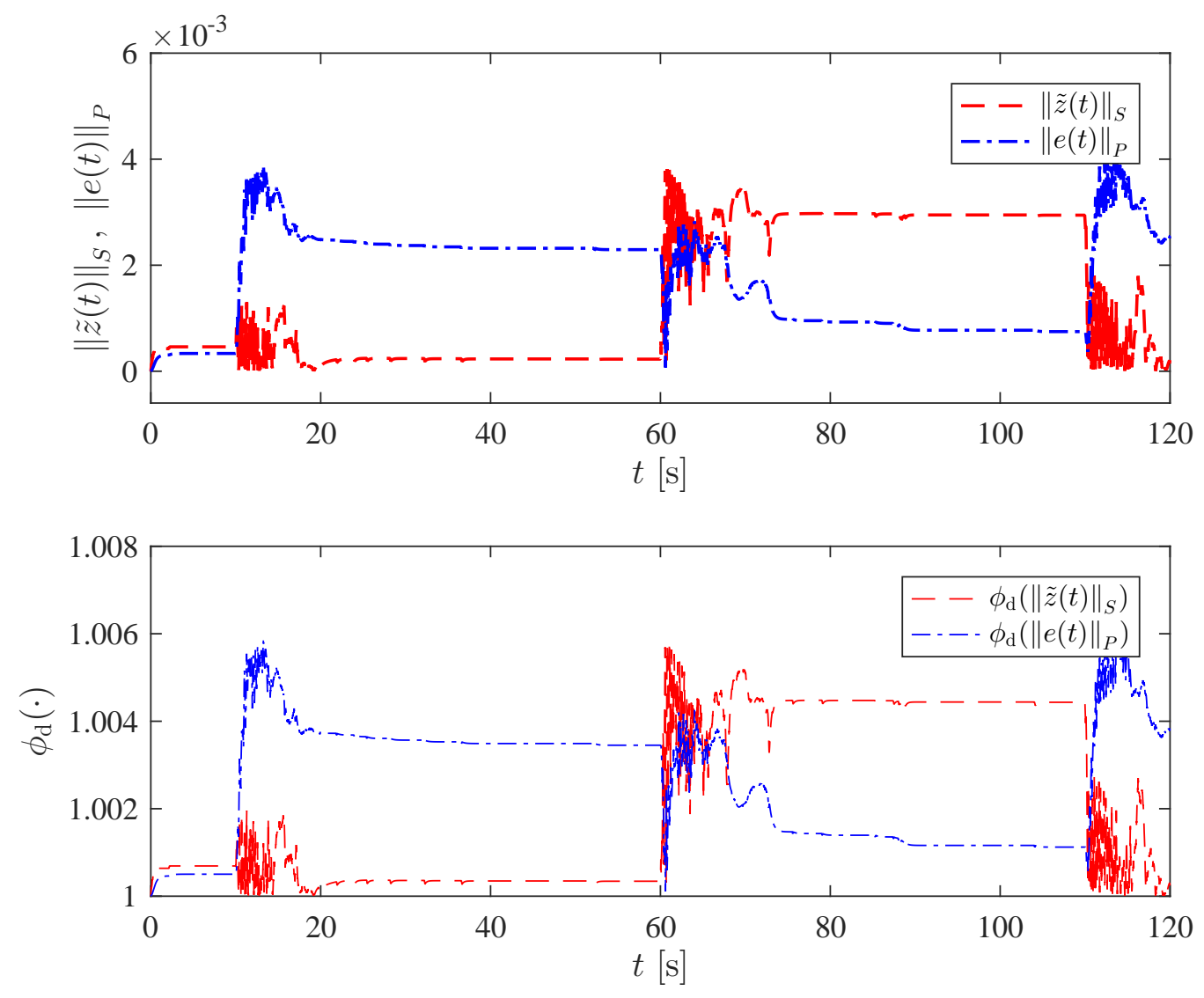

Figure 2.9: Experimental results for proposed set-theoretic adaptive controller (error dependent learning gains and enforced performance bounds for $\varepsilon_{\tilde{z}}=\varepsilon_{e}=0.3$ with artificial disturbance and matched uncertainties). 


\section{Chapter 3: Concluding Remarks and Future Research}

\subsection{Concluding Remarks}

The research reported in this thesis has complemented recent theoretical studies on a proposed adaptive control architecture for an uncertain dynamical system. Through experimental plots, the experimental validation for these studies was shown on a coupled rigid body system with a flexible interconnection link. Specifically, the benchmark mechanical system setup contained an actuated cart coupled with an unactuated cart through a spring in the presence of artificial disturbances, unknown frictions and uncertainties.

Specifically, we used the proposed adaptive control architecture in [1], which is able to individually control and enforce performance bounds on actuated and unactuated dynamics, respectively. Using a settheoretic adaptive control based on restricted potential functions, the interconnected system obtained the stability and enforced performance guarantees. In the presence of both unknown physical interconnections and uncertainties, the system error trajectories for the actuated and unactuated dynamics, respectively, were restricted to user-defined limits. These observations coincide with the simulated results in [1]. Experimental plots showcasing the adaptive controller are provided to show the efficacy of the proposed adaptive control architecture.

\subsection{Future Research}

In this section, we consider some research directions and suggestions for future work related to the results in this thesis. In addition to the presented experiment in Chapter 2, additional experimentation with an actuated cart and an unactuated cart including a pendulum could be conducted in order to further show the effectiveness of the proposed adaptive control architecture. More complex systems can also be considered (i.e., additional carts) to help bridge the gap between theory and application. 


\section{References}

[1] B. C. Gruenwald, T. Yucelen, and A. Chakravarthy, "On adaptive control of unactuated dynamical systems through interconnections with stability and performance guarantees," IEEE Conference on Decision and Control, pp. 595-600, 2018.

[2] T. Yucelen, "Model reference adaptive control," in Wiley Encyclopedia of Electrical and Electronics Engineering (J. G. Webster, ed.).

[3] K. A. Cespedes, K. M. Dogan, E. Yildirim, B. C. Gruenwald, T. Yucelen, and C. Animesh, "Experimental results of a model reference adaptive control approach on an interconnected uncertain dynamical system," in AIAA Guidance, Navigation, and Control Conference, 2020.

[4] E. Arabi, B. C. Gruenwald, T. Yucelen, and N. T. Nguyen, "A set-theoretic model reference adaptive control architecture for disturbance rejection and uncertainty suppression with strict performance guarantees," International Journal of Control, vol. 91, pp. 1195-1208, 012018.

[5] Quanser IP02 Linear Inverted Pendulum User Manual, 2012. Available at https://www.quanser.com/products/linear-servo-base-unit-inverted-pendulum/.

[6] E. Lavretsky and K. Wise, Robust and adaptive control with aerospace applications. Springer, 2013.

[7] H. K. Khalil, "Adaptive output feedback control of nonlinear systems represented by input-output models," IEEE Transactions on Automatic Control, vol. 41, no. 2, pp. 177-188, 1996. 


\section{Appendix A: Projection Operators}

We use the following (rectangular) projection operator definition from [Exercise 11.3, [6]] and [[7]] in this paper.

Definition A.I: Consider a convex hypercube in the form $\Omega=\left\{\theta \in \mathbb{R}^{n}:\left(\theta_{i}^{\min } \leq \theta_{i} \leq \theta_{i}^{\max }\right)_{i=1,2, \cdots, n}\right\}$, where $\Omega \in \mathbb{R}^{n}$, and $\theta_{i}^{\min }$ and $\theta_{i}^{\max }$ respectively represent the minimum and maximum bounds for the $i^{\text {th }}$ component of the $n$-dimensional parameter vector $\theta$ (we set $\theta_{i}^{\min }=-\theta_{i}^{\max }$ for the results of this paper and without loss of generality). Furthermore, for a sufficiently small positive constant $\varepsilon_{0}$, consider another hypercube in the form $\Omega_{\varepsilon_{0}}=\left\{\theta \in \mathbb{R}^{n}:\left(\theta_{i}^{\min }+\varepsilon_{0} \leq \theta_{i} \leq \theta_{i}^{\max }-\varepsilon_{0}\right)_{i=1,2, \cdots, n}\right\}$, where $\Omega_{\varepsilon_{0}} \subset \Omega$. The projection operator Proj: $\mathbb{R}^{n} \times \mathbb{R}^{n} \rightarrow \mathbb{R}^{n}$ is then defined component-wise by $\operatorname{Proj}(\theta, y)=\left(\theta_{i}^{\max }-\theta_{i}\right) y_{i} / \varepsilon_{0}$ if $\theta_{i}>\theta_{i}^{\max }-\varepsilon_{0}$ and $y_{i}>0, \operatorname{Proj}(\theta, y)=\left(\theta_{i}-\theta_{i}^{\min }\right) y_{i} / \varepsilon_{0}$ if $\theta_{i}<\theta_{i}^{\min }+\varepsilon_{0}$ and $y_{i}<0$, and $\operatorname{Proj}(\theta, y)=y_{i}$ otherwise, where $y \in \mathbb{R}^{n}$.

Based on the above definition and $\theta^{*} \in \Omega_{\varepsilon_{0}}$, one can show that the inequality $\left(\theta-\theta^{*}\right)^{\mathrm{T}}(\operatorname{Proj}(\theta, y)-$ $y) \leq 0$ holds for $\theta \in \Omega$ and $y \in \mathbb{R}^{n}$. Moreover, the above definition can be similarly generalized to matrices as $\operatorname{Proj}_{\mathrm{m}}(\Theta, Y)=\left(\operatorname{Proj}\left(\operatorname{col}_{1}(\Theta), \operatorname{col}_{1}(Y)\right), \ldots, \operatorname{Proj}\left(\operatorname{col}_{m}(\Theta), \operatorname{col}_{m}(Y)\right)\right.$ with the inequality $\operatorname{tr}[(\Theta-$ $\left.\left.\Theta^{*}\right)^{\mathrm{T}}\left(\operatorname{Proj}_{\mathrm{m}}(\Theta, Y)-Y\right)\right]=\sum_{i=1}^{m}\left[\operatorname{col}_{i}\left(\Theta-\Theta^{*}\right)^{\mathrm{T}}\left(\operatorname{Proj}\left(\operatorname{col}_{i}(\Theta), \operatorname{col}_{i}(Y)\right)-\operatorname{col}_{i}(Y)\right)\right] \leq 0$ for $n \times m$ matrices $Y$, $\Theta$, and $\Theta^{*}\left(\right.$ here, $\operatorname{col}_{i}(\cdot)$ denotes $i$ th column function). 